\title{
Morvan syndrome
}

INSERM

\section{Source}

INSERM. (1999). Orphanet: an online rare disease and orphan drug data base. Morvan syndrome. ORPHA:83467

Morvan syndrome is a rare, life-threatening, acquired neurologic disease characterized by neuromyotonia, dysautonomia and encephalopathy with severe insomnia. Signs involving central (e.g. hallucinations, confusion, amnesia, myoclonus), autonomic (e.g. variations in blood pressure, hyperhidrosis) and peripheral (e.g. painful cramps, myokymia) hyperactivity, as well as systemic manifestations (such as weight loss, pruritus, fever), are reported. Thymoma is present in some cases. 